\title{
A Genetic Algorithm Approach to Design Principles for Organic Photovoltaic Materials
}

de Sousa, Leonardo Evaristo; da Silva Filho, Demétrio Antônio; de Silva, Piotr; Ribeiro, Luciano; de Oliveira Neto, Pedro Henrique

Published in:

Advanced Theory and Simulations

Link to article, DOI:

10.1002/adts.202000042

Publication date:

2020

Document Version

Peer reviewed version

Link back to DTU Orbit

Citation (APA):

de Sousa, L. E., da Silva Filho, D. A., de Silva, P., Ribeiro, L., \& de Oliveira Neto, P. H. (2020). A Genetic Algorithm Approach to Design Principles for Organic Photovoltaic Materials. Advanced Theory and Simulations, 3(8), [2000042]. https://doi.org/10.1002/adts.202000042

\section{General rights}

Copyright and moral rights for the publications made accessible in the public portal are retained by the authors and/or other copyright owners and it is a condition of accessing publications that users recognise and abide by the legal requirements associated with these rights.

- Users may download and print one copy of any publication from the public portal for the purpose of private study or research.

- You may not further distribute the material or use it for any profit-making activity or commercial gain

- You may freely distribute the URL identifying the publication in the public portal 


\section{A Genetic Algorithm Approach to Design Principles for Organic Photovoltaic Materials}

\section{Leonardo Evaristo de Sousa ${ }^{1}$ | Demétrio Antônio da Silva Filho $^{4,2}$ | Piotr de Silva ${ }^{3}$ | Luciano Ribeiro ${ }^{1}$ | Pedro Henrique de Oliveira Neto ${ }^{2,}$}

\footnotetext{
${ }^{1}$ Theoretical and Structural Chemistry Group, State University of Goias, Anapolis, Brazil

${ }^{2}$ Institute of Physics, University of Brasilia, Brasilia, 70919-970 - Brasilia, Brazil

${ }^{3}$ Department of Energy Conversion and Storage,Technical University of Denmark, Anker Engelunds Vej 301, 2800 Kongens Lyngby

${ }^{4}$ Institute for Advanced Studies, University of Cergy-Pontoise, 1 rue Descartes, 95000 , Neuville-sur-Oise, France

\section{Correspondence}

Pedro Henrique de Oliveira Neto, Institute of Physics, University of Brasilia, Brasilia, 70919-970, Brasilia, Brazil Email: pedrohenrique@unb.br

Funding information
}

The increase in the efficiency of organic photovoltaic (OPV) devices relies on understanding the underlying science of several interconnected physical mechanisms that prevent one-dimensional optimization strategies to succeed. Here, we employ a combination of Kinetic Monte Carlo simulations of exciton dynamics with a Genetic Algorithm to automatically optimize the external quantum efficiency of donor-acceptor interfaces under different scenarios. Simulations include phenomena from light absorption to exciton diffusion, dissociation, radiative recombination, and internal conversion, thus modeling the main physical processes that define the overall efficiency of an OPV up to charge separation. We show that when internal conversion is kept in check, the combination of optimal transition dipole moments and absorption energies points at low bandgap polymers as the most promising candidates for donor materials. However, when non-radiative deexcitation mechanisms are stronger, the optimization strategy shifts towards higher bandgaps, focusing rather on increasing the fluorescence quantum yield of the donor. Finally, our approach shows that adjusting the energy levels of the acceptor so that exciton transfers across the interface become negligible produces important gains in efficiency and at the same time reduces the system's dependence on large electronic couplings. Our findings indicate pathways for the engineering of highly efficient organic interfaces. 


\section{1 | INTRODUCTION}

Research in organic photovoltaic (OPV) devices has been struggling in the past years against the challenges of increasing device efficiency and stability as well as scaling up production so that this technology may reach economic viability ${ }^{[1,2,3]}$. Efforts in this sense require knowledge of the mechanisms involved in the working of such devices, so that optimization criteria may be developed $^{[4,5]}$. These mechanisms go from the absorption of light through the diffusion and dissociation of excitons at donor-acceptor interfaces to finally electron hole separation and charge collection ${ }^{[6]}$.

Optimization strategies start by tuning the absorption spectrum of the molecules responsible for harvesting light. This tuning comprises of choosing molecules with optical gaps consistent with the peak of the solar spectrum. The absorption of light, in its turn, results in the generation of excitons in the material, which are quasiparticles composed of electron-hole pairs bound by Coulomb interaction. Because of the low dielectric permittivity of organic materials, exciton binding energies for organics are of the order of hundreds of $\mathrm{meV}^{[7]}$, well above typical values for thermal energy. As such, excitons will hardly be able to spontaneously dissociate in the bulk of an organic material, rather having to diffuse until they reach a donor-acceptor interface.

The diffusion of singlet excitons results mainly from a non-radiative process known as the Förster resonance energy transfer ${ }^{[8,9,10]}$. In this process, energy is transferred between neighboring molecules provided there is overlap between their absorption and emission spectra. The efficiency with which this process takes place is often quantified by a characteristic distance termed the Förster radius, which is the distance at which the probability of exciton transfer equals the probability of radiative exciton recombination. As such, large Förster radii are crucial to device efficiency as they control the exciton diffusion length and consequently the amount of excitons able to reach donor-acceptor interfaces. This puts constraints on molecular design as the necessary spectral overlap depends on factors such as the molecule's optical gap, its reorganization energy and its transition dipole moment. In addition, exciton diffusion also competes against non-radiative deexcitation pathways that arise, for instance, as a result of internal conversion. In this process, singlet excitons decay to the ground state non-radiatively due to the vibronic coupling between ground and excited state wave functions $^{[11]}$. Non-radiative decay mechanisms are known to limit exciton lifetimes ${ }^{[12]}$ and constitute a source of inefficiency in organic photovoltaic devices ${ }^{[13]}$ which must be taken into account when considering design principles.

Finally, the actual mechanism of charge transfer requires a non-zero electronic coupling between an excited donor molecule and a ground state acceptor. In addition, for this process to take place efficiently it must be energetically favorable, with a driving force strong enough to overcome the exciton binding energy and push the creation of a charge transfer state ${ }^{[14,2,15]}$. This driving force is achieved by judiciously choosing donor and acceptor molecules in such a way as to produce the appropriate energy difference between their frontier orbitals. For instance, a donor excited molecule with an energy level higher than that of an adjacent acceptor favors the dissociation of excitons by means of electron transfers to the acceptor. The importance of such pairing of energy levels has also been shown to relate to the open circuit voltage and thus to the overall efficiency of the photovoltaic cell ${ }^{[16]}$.Furthermore, morphology plays a vital role in the efficiency of charge transfer and efforts regarding morphology tuning have been undertaken by several means including reducing surface roughness ${ }^{[17]}$, application of electric fields ${ }^{[18]}$, different choices of solvents and additives ${ }^{[19,20,21]}$ and molecular engineering ${ }^{[22,23]}$

When it comes to modeling the various phenomena involved in the working of an OPV device, many studies have focused on each individual process at play. For instance, electronic structure methods have been employed in the simulation of absorption and emission spectra ${ }^{[24,25,26,27]}$ and on the prediction of exciton diffusion lengths ${ }^{[28,29]}$. Exciton diffusion $^{[30,31,32,33]}$, charge transport ${ }^{[34,35,36]}$ and charge recombination ${ }^{[37,38]}$ have also been studied individu- 
ally as have the effects of morphology on device efficiency ${ }^{[39]}$. As it may be inferred from this discussion, the simultaneous consideration of all these processes in a simulation would require many parameters, testifying to the complexity of the mechanisms under study. The question then becomes what combination of parameters may produce simulations for which the amount of exciton dissociation is maximum. However, because of the interdependence between the different physical mechanisms, the answer to this question is not straightforward. For instance, large conjugation lengths may result in large transition dipole moments which, in one hand, improve the efficiency of exciton transfer by increasing the Förster radius. At the same time, it may also hinder exciton diffusion by increasing the effective hopping distance of excitons ${ }^{[40]}$. Also, in spite of the necessity of efficient exciton diffusion for device performance, this very process may constitute a competing factor against charge transfer either by allowing excitons to hop across the donor-acceptor interface or by merely contributing to entropy effects against exciton dissociation ${ }^{[41,42]}$. In addition, reducing a material's energy gap to match the solar spectrum increases the amount of absorbed photons, but also increases the rate of non-radiative deexcitation. Finally, small donor domains reduce the distance that excitons must travel before reaching the interface, but at the same time reduce the amount of absorbed photons. In order to find an optimal solution that takes into account all these aspects, a multi-dimensional strategy for determining the parameter values that optimize exciton dissociation is required.

Considering all the aforementioned mechanisms and the relationships between them, it becomes clear that in order for consistent optimization strategies to be developed, it is necessary to model the process in as complete a manner as possible. In this work, we develop a model to simultaneously simulate absorption and emission spectra as well as exciton diffusion, recombination, and dissociation into charge transfer (CT) states. The model focuses on interconnected phenomena whose optimization is not straightforward and consists of a Kinetic Monte Carlo (KMC) simulation in which excitons must diffuse through a lattice of donor sites that is sur- rounded by acceptor sites. Excitons may diffuse, recombine either radiatively or by internal conversion or dissociate into CT states. The model reduces such complex phenomena to a set of key parameters while taking into consideration the relationships between all of them. To understand how to maximize the amount of excitons that dissociate at the donor-acceptor interface in this scenario of highly interdependent features, we resort to a genetic algorithm (GA). This algorithm searches for the set of parameter values that maximizes the external quantum efficiency (EQE) of the system. GAs have already been successfully employed in the context of physical chemistry ${ }^{[43,44,45]}$, in particular regarding exciton dynamics ${ }^{[46]}$.

Our results show that if non-radiative losses can be kept in check, low bandgap polymers are predicted to produce the most efficient donor materials. As nonradiative rates increase, however, the optimal donor features shift towards higher bandgap materials, with a focus on increasing fluorescence quantum yields. In addition, we show that the elimination of competing processes at the interface, such as exciton transfers from donor to acceptor, constitute a simple measure that is able to increase optimal internal quantum efficiency (IQE) in around $10 \%$, while simultaneously reducing the dependence of device efficiency on large electronic couplings, which are more difficult to engineer properly. The results presented here provide clear principles for rational design of devices.

\section{2 | METHODS}

\subsection{The Kinetic Monte Carlo Model}

Kinetic Monte Carlo simulations are run on a cubic lattice composed of donor sites. Acceptor sites are positioned at the edges of the lattice, making the donoracceptor interface (Figure 1-a). Each site in the simulation is assigned a LUMO (Lowest Unoccupied Molecular Orbital) energy level and a bandgap value $g$ drawn from a gaussian distribution with a given mean and variance $\sigma^{2}$, allowing for variations in energy levels while preserving the correlations in $\mathrm{HOMO}$ (Highest Occupied Molec- 


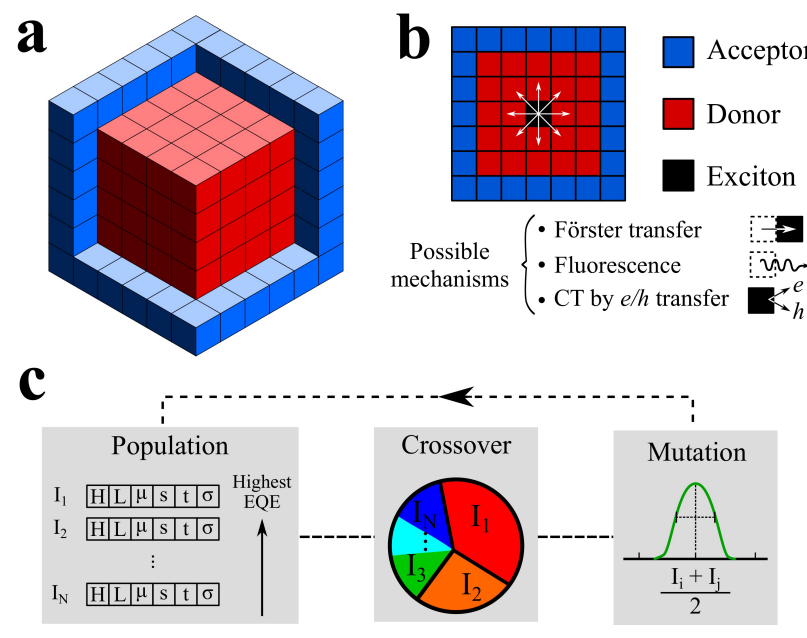

FIGURE 1 Schematics of the Genetic Algorithm-Kinetic Monte Carlo model. a) Morphology employed in the model. A cubic lattice of donor sites (red) enclosed by acceptor sites (blue). b) Cross section of the architecture shown in a. Excitons (black) are generated at a distance from the donor-acceptor interface. Four processes - Förster transfer, exciton recombination and electron and hole transfers - compete in defining the behavior of the exciton at each step. c) Representation of the genetic algorithm showing the ranking of individuals based on EQE, the crossover that generates new individuals and the mutation process that increases variability.

ular Orbital) and LUMO changes ${ }^{[47]}$. The ensuing distribution of energy levels in the system produces absorption and emission spectra with a variance of $\sigma^{2}$. This makes the connection between energetic disorder and spectral broadness and also allows us to calculate absorption and emission spectra.

Single band absorption cross-sections $(\alpha)$ and differential emission rates $(I)$ are computed as ${ }^{[48,26,24]}$

$$
\alpha(\omega)=\frac{\pi e^{2} \hbar}{2 m c \epsilon_{0}} f_{01} G\left(\hbar \omega-\Delta E_{01}, \sigma\right),
$$

and

$$
I(\omega)=\frac{e^{2}}{2 \pi \hbar m c^{3} \epsilon_{0}} \Delta E_{10}^{2}\left|f_{10}\right| G\left(\hbar \omega-\Delta E_{10}, \sigma\right),
$$

$e$ and $m$ being the electron charge and mass, respectively, $\epsilon_{0}$ the vacuum permittivity, $c$ the speed of light and $\omega$ the radiation frequency. The optical gap is denoted by $\Delta E_{i j}$ and $G$ is a gaussian function with standard deviation $\sigma$. The term $f_{i j}$ is the oscillator strength of the transition, given by

$$
f_{i j}=\frac{2 m \Delta E_{i j} \mu^{2}}{3 \hbar^{2} e^{2}}
$$

where $\mu$ is the transition dipole moment.

In the case of absorption, the optical gap may be calculated as

$$
\Delta E_{01}=E_{L U M O}-E_{H O M O}-X_{b e}
$$

with $X_{b e}$ representing the exciton binding energy. The fluorescence energy differs from the absorption energy by the Stokes' shift, which may be approximated as two times the molecular reorganization energy ${ }^{[49]}(\lambda)$, yielding

$$
\Delta E_{10}=\Delta E_{01}-2 \lambda
$$

In this sense, the absorption and emission spectra are determined in our model by the HOMO and LUMO 
energy levels, the transition dipole moment, the exciton binding energy and the molecular reorganization energy.

The solar emission spectrum is modeled by the black body radiation spectrum at temperature $\mathrm{T}=5778 \mathrm{~K}^{[50]}$. By matching the absorption cross-section with the solar spectrum and taking into account attenuation effects associated with lattice size, we calculate the proportion of generated excitons in a given system as

$$
N_{e x}=\frac{n_{a b s}}{N_{0}}=\int_{0}^{\infty}[1-\exp (-n \alpha(\omega) L)] d \omega
$$

where $n_{a b s}$ is the number of absorbed photons, $N_{0}$ corresponds to the total number of photons emitted (set at $10^{4}$ in our simulations), $L$ is the system's width and $n$ is the number of absorbing molecules per unit volume.

At the beginning of a simulation, excitons are generated in the middle of the lattice and, at each time step, may hop to neighboring sites through a Förster transfer, recombine either radiatively or non-radiatively, or dissociate into a CT state by means of an electron or hole transfer to a nearby site (Figure 1-b). The energetic disorder of the system is taken into account for all transfer mechanisms. The selection of the actual process that takes place is done by a weighted random selection algorithm that is repeated until all excitons have either recombined or dissociated.

The rate of exciton transfer is calculated as ${ }^{[8,10]}$

$$
k_{F}=\frac{1}{\tau_{r a d}}\left(\frac{R_{F}}{\beta \mu+r}\right)^{6} e^{-\left(\Delta E_{01}+\left|\Delta E_{01}\right|\right) / 2 k T},
$$

where $r$ represents intermolecular distances, $R_{F}$ is the Förster radius and $\tau_{\text {rad }}$ is the donor's radiative lifetime. The above expression includes effects stemming from energetic disorder by multiplying the Förster rate by a Boltzmann factor ${ }^{[51]}$. It also takes into account a correction term for short distances, given by $\beta \mu^{[40,52]}$, in which $\beta=1.15 e^{-1}$ is a constant that multiplies the transition dipole moment. Furthermore, these intermolecular distances are written as a function of molecular size by $(r=5+\beta \mu / 2) \AA$. The $\beta \mu / 2$ term serves as a proxy for molecular conjugation length and accounts for the fact that for larger molecules, distances between molecular centers of mass should increase.

The Förster radius and radiative lifetime are obtained from the absorption and photoluminescence spectra using

$$
R_{F}^{6}=\frac{9 c^{4} \kappa^{2} \tau_{r a d}}{8 \pi} \int_{0}^{\infty} \frac{d \omega}{\omega^{4}} I_{D}(\omega) \alpha_{A}(\omega),
$$

where $\kappa$ is an orientation factor whose average for random molecular orientations is $2 / 3^{[53]}$, and

$$
\frac{1}{\tau_{r a d}}=\int_{0}^{\infty} I_{D}(\omega) d \omega
$$

Charge transfer rates are calculated with the Marcus equation ${ }^{[54]}$

$$
k_{C T}=t^{2} \sqrt{\frac{\pi}{\hbar^{2} \lambda k T}} e^{-\frac{(\lambda+\Delta G)^{2}}{4 \lambda k T}}
$$

in which $\Delta G$ is the difference in Gibbs free energy, which may be approximated by site energy differences if entropy contributions may be neglected, and $t$ is the electronic coupling. It is worth noting that $t$ is the coupling $\langle D(S 1) A(S 0)|H| D( \pm) A(\mp)\rangle$ between an initial state composed of a neutral excited donor $(\mathrm{D}(\mathrm{S} 1))$ and a ground state acceptor $(\mathrm{A}(\mathrm{SO}))$ and a final state composed of oppositely charged molecules $(D( \pm)$ and $A(\mp))$, corresponding to either an electron or hole transfer. This coupling may differ importantly from the usual transfer integral from charged to neutral molecules that is relevant to charge transfer $(\langle D( \pm) A(S 0)|H| D(S 0) A(\mp)\rangle)^{[14]}$.

Finally, the rate of internal conversion follows the energy gap law ${ }^{[11]}$

$$
k_{n r}=k_{0} \exp \left(-\frac{\gamma \Delta E_{01}}{\hbar \omega_{M}}\right)
$$

in which the $\gamma$ constant is given by $\gamma=\left[\ln \left(\Delta E_{01} / \lambda\right)-1\right]$, the pre-exponential factor $k_{0}$ is a constant proportional to the square of the molecular vibronic coupling, $\Delta E_{01}$ is the optical gap and $\omega_{M}$ is the highest normal mode frequency of the molecule, which is taken as $\approx 3200 \mathrm{~cm}^{-1}$, corresponding to $\mathrm{C}-\mathrm{H}$ stretching modes ${ }^{[11]}$. 


\section{2 | The Genetic Algorithm}

\begin{tabular}{|l|l|l}
\hline Feature & Donor & Acceptor \\
\hline \hline LUMO & gene & $-3.7 \mathrm{eV}$ \\
\hline Gap & gene & $2.4 \mathrm{eV}$ \\
\hline$\mu$ & gene & $1.0 \mathrm{a.u}$. \\
\hline $\mathrm{t}$ & gene & - \\
\hline Lattice Size & gene & - \\
\hline$\sigma$ & gene & $0.15 \mathrm{eV}$ \\
\hline$X_{b e}$ & $0.3 \mathrm{eV}$ & $0.3 \mathrm{eV}$ \\
\hline$\lambda$ & $0.3 \mathrm{eV}$ & $0.3 \mathrm{eV}$ \\
\hline
\end{tabular}

TABLE 1 List of model parameters characterizing the donor and acceptor materials. Genes are the features that are subject to optimization.

Model parameters are presented in Table 1 divided into two categories: one for the donor and another for the acceptor material. The first category includes the parameters that the genetic algorithm seeks to optimize (the genes). These are the donor's LUMO and bandgap $g$, its transition dipole moment $\mu$, the electronic coupling $t$ associated with exciton dissociation, the number of sites in each side of the cubic lattice and the standard deviation of the absorption and emission spectra $\sigma$. In addition, the donor's exciton binding energy and reorganization energy are kept constant as they are not subject to optimization, rather being constraints. Both are set to $0.3 \mathrm{eV}$, corresponding to typical values found in $\pi$-conjugated molecules ${ }^{[5,56]}$. The second category shown in Table 1 refers to the acceptor material, whose parameters were chosen so as to represent PCBM, which is a widely used electron acceptor molecule ${ }^{[57,58,59,60]}$, and were kept constant throughout all simulations presented here unless stated otherwise. Finally, all simulations were run at $300 \mathrm{~K}$ and the optimization algorithm was run in several scenarios to evaluate the differences in optimization strategy.

The genetic algorithm is schematically shown in Figure 1-c. A set of genes is said to characterize an indi- vidual in the sense that a set of parameter values determines a particular KMC simulation. The process begins with the generation of 30 such individuals, each with randomly selected values for its genes. The resulting KMC simulations are run each with 200 excitons and once they are over, the ratio of excitons that dissociated by means of electron transfers to acceptor sites is registered. This number is multiplied by the proportion of generated excitons $\mathrm{N}_{e x}$. This provides a measure of the EQE of the system and serves as the number that evaluates the performance of a given simulation. It is worth mention that the EQE we compute in this way is not monochromatic, as the computation of $\mathrm{N}_{e x}$ accounts for photons from the whole solar spectrum (see Equation 6).

Once all 30 simulations have been evaluated, a new generation of 30 individuals is produced. Each new individual is created from genes coming from two previous simulations. These two previous simulations are randomly selected from a list containing all simulations that were run in the preceding round of the algorithm in addition to the 10 highest EQE simulations obtained thus far. The probability of a given simulation being chosen as a progenitor for a new individual is proportional to its $E Q E$, so that sets of parameters that present higher performance are more likely to be chosen. With the two progenitors selected, the genes for the new individual are obtained by sampling from a gaussian distribution with mean given by the average value of the progenitors' corresponding genes and a coefficient of variation of 0.3 . This coefficient, given by the ratio between the distribution's mean and standard deviation, effectively controls how powerful mutations are in the algorithm and ensures genetic variation.

The above process is repeated until a thousand generations have been created, at which point thirty thousand configurations will have been evaluated. Each individual KMC simulation is run with 200 excitons each, which provides an appropriate compromise between accuracy and the computational cost necessary for running such number of simulations. 


\section{3 | RESULTS AND DISCUSSION}
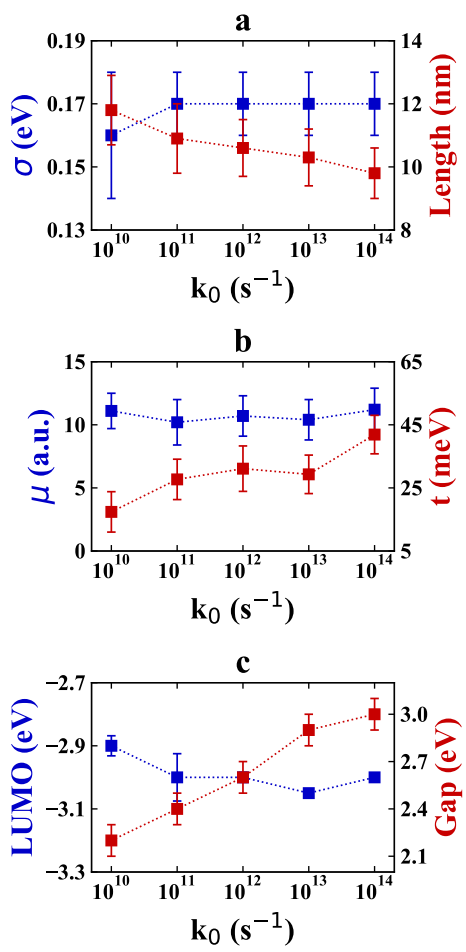

FIGURE 2 Optimization strategy for each model parameter under different vibronic coupling scenarios. Squares represent average values and error bars correspond to standard deviations taken from the ten highest EQE simulations. a) Spectral broadness $(\sigma)$ and system length. b) Transition dipole moment $(\mu)$ and electronic coupling $t$. c) Donor LUMO and bandgap.

We start by addressing the effects that different nonradiative deexcitation rates produce on the optimization strategies by analyzing how the optimization strategy changes for different values of the pre-exponential factor $k_{0}$ (Equation 11). To compare results from different evolution histories, we consider the average and standard deviation of each gene taken from the ten individual simulations with highest EQE obtained in each optimization process. In this context, Figure 2 shows the average optimal values of the genes along with their stan- dard deviation for simulations with $k_{0}=10^{10}-10^{14} \mathrm{~s}^{-1}$, which corresponds to an interval in which this factor has been estimated to lie ${ }^{[11]}$. Results for the spectral broadness $\sigma$ can be seen in Figure 2-a. For most scenarios, an average optimal $\sigma$ of $0.17 \mathrm{eV}$ was obtained. Considering the extremities of the gaussian distribution to be at $\pm 2 \sigma$, this spectrum covers $0.68 \mathrm{eV}$. A broader spectrum would simultaneously increase the number of absorbed photons and the donor-donor Förster radius (because of increased spectral overlap) but the observed evolution does not point in this direction. The reason for this behavior is the energetic disorder such a large spectrum would induce in the system, which would, in turn, hinder exciton diffusion. Thus, the trade-off between increasing the amount of harvested photons by employing a broader spectrum and keeping energetic disorder to a minimum results in the obtained value of $0.17 \mathrm{eV}$. The exception is the case $k_{0}=10^{10} \mathrm{~s}^{-1}$, which shows an average optimal $\sigma$ of $0.16 \mathrm{eV}$, but also shows a larger standard deviation, indicating that lower non-radiative decay rates put less strict constraints in the spectral broadness.

A second optimized feature associated with exciton diffusion is the size of the donor domain. This is also shown in Figure 2-a, where it can be seen that optimum domain sizes decrease continuously from approximately $12 \mathrm{~nm}$ to $10 \mathrm{~nm}$ as $k_{0}$ increases. These values result from the opposing needs of increasing the number of absorbing sites and reducing the distances necessary for excitons to travel before reaching the interface. This latter issue becomes more pressing as the rate of nonradiative decay grows larger, lowering optimal domain sizes. In agreement with experimental findings, these results suggest that $10-12 \mathrm{~nm}$ should be the typical size of donor domains in bulk heterojunctions ${ }^{[61,62,63]}$ or in interdigitated architectures ${ }^{[64]}$ as these sizes provide the best compromise between light harvesting and exciton dissociation efficiency. Besides the donor domain size, efficient light harvesting also profits from large absorption cross-sections, which are proportional to the square of the transition dipole moment $\mu$. The optimization of this feature suffers no significant change in the different scenarios, as shown in Figure 2-b. Optimal av- 
a

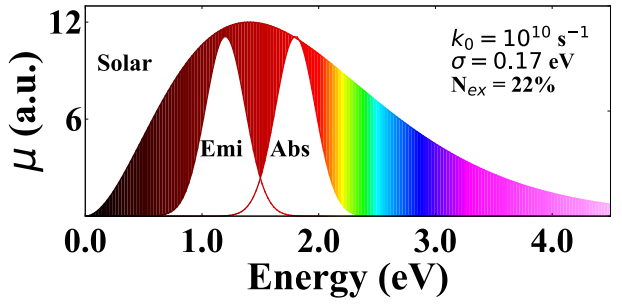

b

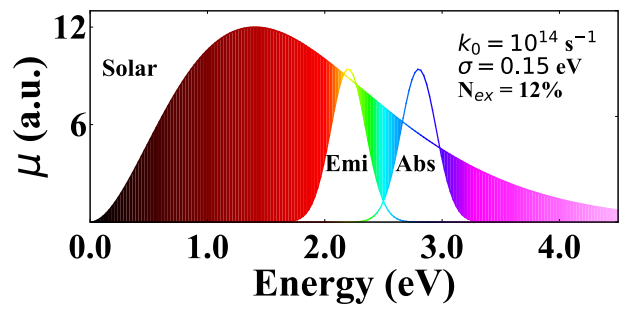

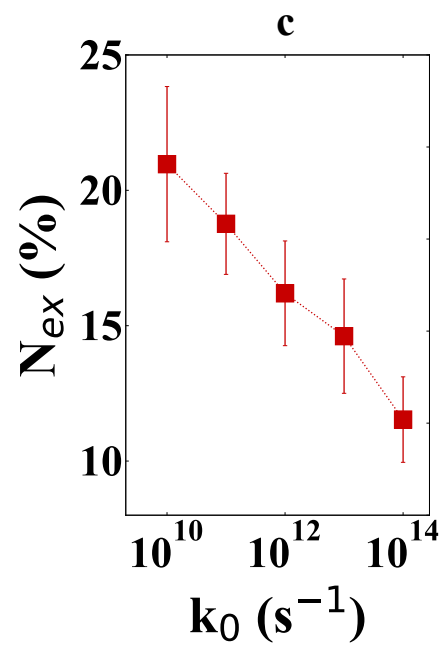

FIGURE 3 Schematics of the optimal absorption and emission spectra found for the scenarios of a) $k_{0}=10^{10} \mathrm{~s}^{-1}$ and $\mathrm{b}$ ) $k_{0}=10^{14} \mathrm{~s}^{-1}$. c) Percentage of generated excitons relative to incident photons $\left(N_{e x}\right)$ as a function of $k_{0}$.

erage values range from 10 to 12 a.u., which are associated with large conjugation lengths often found in $\pi$ conjugated polymers. Large values of $\mu$ also translate into higher differential emission rates and consequently on higher Förster transfer rates due to increased spectral overlap, favoring exciton diffusion. On the other hand, optimal $\mu$ values do not increase indefinitely, as they are also bounded by their impact on the effective intermolecular distances that negatively affect the exciton transfer rates.

The next gene shown in Figure 2-b is the electronic coupling $t$. For $k_{0}=10^{10} \mathrm{~s}^{-1}$, an average optimal coupling of $17 \mathrm{meV}$ was found. As larger non-radiative decay rates are employed, the optimal couplings are pushed to higher values, reaching up to $42 \mathrm{meV}$. In the model, the optimization of electronic couplings reflects a necessary balance between expediting exciton dissociation at the interface and preventing the spontaneous dissociation of excitons in the bulk, which could be driven by energetic disorder. The changes in optimal average electronic coupling are also associated with the optimization strategy of the electronic levels of the donor species. This is highlighted in Figure 2-c, which shows the optimal donor LUMO and gap for each scenario. LUMO optimization produces nearly the same results in all scenarios, with an average value $-3.0 \mathrm{eV}$. An average donor LUMO value of $-3.0 \mathrm{eV}$ in combination with an average acceptor LUMO of $-3.7 \mathrm{eV}$ results in a driving force $\Delta G=-0.7 \mathrm{eV}$. The maximum transfer rate is obtained when $\Delta G+\lambda=0$ (see Equation $10)$, which in the case of our simulations would require $\Delta G=-0.3 \mathrm{eV}$. Interestingly, this value was not found. This is so because it does not account for the presence of energetic disorder, which makes optimization of CT rates not as straightforward. Since excitons tend to hop towards lower energy sites, donor sites that excitons actually reach at the interface should present lower energy levels. As such, it is likely that at the interface, the CT rates are much closer to maximum levels. This is further corroborated by the fact that energy disorder with standard deviation of $0.17 \mathrm{eV}$ was present in most cases, which shows that a $-0.34 \mathrm{eV}$ decrease with respect to the average in LUMO energies corresponds to two standard deviations, indicating that excitons that reach the 
interface are approaching the tail of the density of states. In this sense, the optimization procedure shows preference for optimizing CT rates at the interface by means of a pairing of donor and acceptor energy levels that accounts for energetic disorder.

Finally, the energy gap's behavior shows the most striking changes in optimization strategy. As the vibronic couplings increase, pushing the $k_{0}$ parameter to larger values, average optimal gaps move continuously upwards from $2.2 \mathrm{eV}$ to $3.0 \mathrm{eV}$. One effect of this increase is seen in the open circuit voltage of each cell, which can be calculated by means of an empirical relationship found between the donor's HOMO and PCBM's LUMO ${ }^{[16]}$. This calculation, detailed in the Supporting Information (SI), indicates that average open circuit voltages of the optimized simulations increase from $0.78 \mathrm{~V}$ to $1.68 \mathrm{~V}$ (see Figure SI-1), following the rise in energy gaps. These values are bound to overestimate actual open circuit voltages due to the neglect of further loss mechanisms, but experimental work has already pointed out the association between wide-gap donor polymers with high open circuit voltages, in line with our estimates $^{[65]}$.

Clearly, this increase in energy gaps is driven by the energy gap law (Equation 11), as a means to reduce the non-radiative decay rates. The main consequence of this change in bandgaps is the progressive blue shift of the absorption spectra. A comparison between the absorption and emission spectra of two simulations of highest EQE found for the $k_{0}=10^{10} \mathrm{~s}^{-1}$ and $k_{0}=$ $10^{14} \mathrm{~s}^{-1}$ can be seen in Figure 3-a and $b$, respectively. Whereas the first shows absorption in the red part of the spectrum, the latter absorbs in the blue region, both with similar spectral broadness. The overlap with the solar spectrum highlights the difference in exciton density expected for each case. We see that the optimization strategy in the case of milder vibronic coupling aims at maximizing the number of absorbed photons by approaching the absorption peak to the solar spectrum peak, which results in the proportion of incident photons generating excitons reach $\mathrm{N}_{e x}=22 \%$. The combination of large transition dipole moments with bandgaps around $2.0 \mathrm{eV}$ points at low bandgap poly- mers as candidates to fill the role of optimum electron donors in organic photovoltaic devices for which nonradiative mechanisms can be kept in check. There are several examples of such polymers ${ }^{[66]}$ and recently new ones have been developed and applied to organic solar cells with expressive results $[67,68,69,70,71,72,73]$. On the other hand, when internal conversion is strong, the optimization strategy shifts to higher bandgaps, moving the absorption spectrum away from the solar peak, as seen in Figure 3-b for the highest EQE simulation in the $k_{0}=10^{10} \mathrm{~s}^{-1}$ scenario. As a consequence of this spectral shift, the number of generated excitons decreases to $\mathrm{N}_{e x}=12 \%$. Figure 3-c shows that the average $\mathrm{N}_{e x}$ number drops from $21 \% \pm 3 \%$ to $12 \% \pm 2 \%$ when going from the smallest to largest $k_{0}$ values. Considering that optimal transition dipole moments and spectral broadness are nearly the same for all scenarios, the observed decrease in the number of generated excitons can be attributed to the rising optimal bandgaps and declining optimal donor domain sizes. These results reinforce the idea that the success of low bandgap polymers as donor materials is highly contingent on the capacity to minimize non-radiative deexcitation pathways.

The effects of these different optimization strategies are elucidated when we look into the efficiency of the top ten simulations from each case considered here. Figures 4- $a$ and $b$ show the average external and internal quantum efficiencies (EQE and IQE, respectively) for the top ten simulations found in each scenario along with the corresponding standard deviation. We observe that EQEs for the simulations run with different $k_{0}$ more than halve when going from $k_{0}=10^{10} \mathrm{~s}^{-1}$ to $k_{0}=10^{14} \mathrm{~s}^{-1}$, which correlates well with the observed decrease in the number of generated excitons. On the other hand, Figure 4-b shows a less steep decrease in IQEs, which drop from $50 \%$ to $40 \%$. We see that the more limited EQE observed for simulations with larger $k_{0}$ requires the system to improve its IQE, in order to partially offset the sacrifice in exciton generation rate. Importantly, the EQE estimates reported here are calculated by taking into account the total number of incident photons $\left(10^{4}\right)$ that is distributed over the whole solar spectrum, not only those in the range of the donor's absorption spectrum as 
a

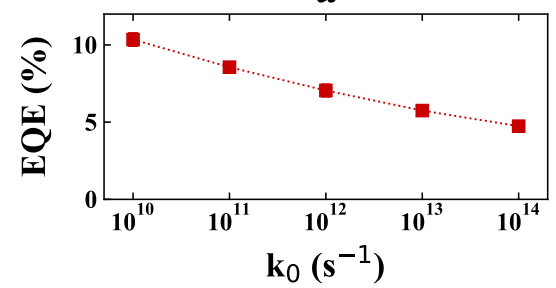

c

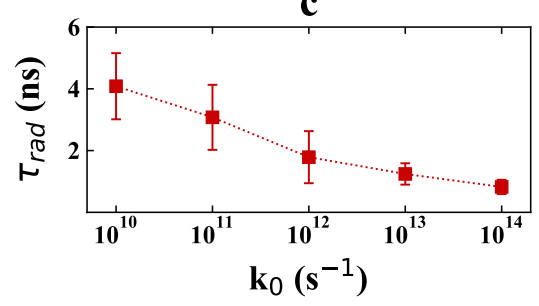

b

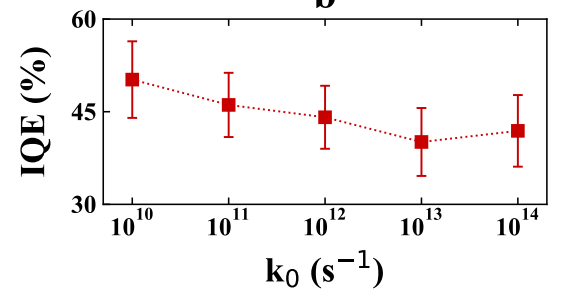

d

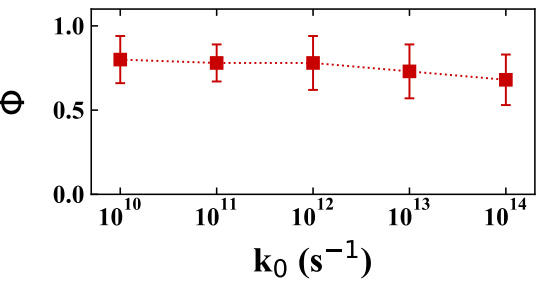

FIGURE 4 Average and standard deviation of several features taken from the top ten simulations under each vibronic coupling scenario. a) EQE, b) IQE, c) exciton radiative lifetime and d) donor fluorescence quantum yield.

usually done in other works. With the optimization strategy often resorting to narrow absorption spectra and small donor domains, a considerable amount of the $10^{4}$ photons is not absorbed, which results in apparently low efficiency values when compared to values usually reported to real photovoltaic devices. On the other hand, IQEs suffer from the presence of the various competing processes that result in exciton quenching, among which the non-radiative deexcitation pathways.

Considering these results, what can explain the shift from lower to higher optimal bandgaps in the simulations with higher non-radiative decay rates if this has been established to reduce the amount of absorbed photons? The answer lies in the data presented in Figure 4-c, which shows the average radiative lifetimes for the top ten simulations in the different scenarios. It can be seen that as $k_{0}$ increases, the optimization process selects configurations that produce lower average radiative lifetimes and lower variance in lifetimes as well, as evidenced by the diminishing error bars. These lower lifetimes are obtained by both higher energy emission peaks $\left(1 / \tau_{\text {rad }} \propto \Delta E_{10}^{3}\right)$ and higher transition dipole moments $\left(1 / \tau_{\text {rad }} \propto \mu^{2}\right)$, as seen in Equation 2. Non- radiative decay mechanisms are known to limit exciton lifetimes, so it becomes clear that when strong internal conversion is enforced, the evolution process moves towards simulations in which the radiative lifetimes become closer to actual exciton lifetimes. This amounts to saying that the optimization strategy selects donor materials that present higher fluorescence quantum yields $\left(\Phi=\tau / \tau_{\text {rad }}\right)^{[53]}$. This can be seen in Figure 4-d, which shows that average quantum yields for the top ten simulations range from 0.80 to 0.68 as the rate of internal conversion rises in spite of the much steeper decay in EQE. This explains why the top ten simulations for larger $k_{0}$ present a higher bandgap as it blue shifts the emission peak energy, reducing $\tau_{\text {rad }}$. It constitutes a sacrifice of the exciton generation rate in favor of higher quantum yield. Interestingly, this result suggests that low exciton lifetimes, which are often mentioned as the most important limiting factor to organic OPVs efficiency, must be countered by higher bandgaps, which simultaneously reduce non-radiative losses and radiative lifetimes, helping keep quantum yields higher. These results highlight the importance of the fluorescence quantum yield as a figure of merit not only in applications devoted to light 
emission but also in the case of photovoltaics. Similar conclusions have been drawn in recent experimental works that reported the development of devices with good photovoltaic performance ${ }^{[4,74]}$.
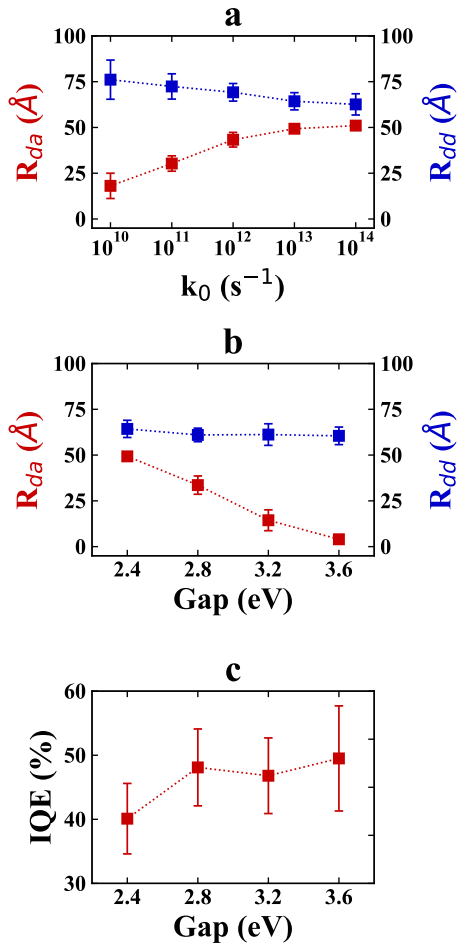

FIGURE 5 a) Average and standard deviations of optimal a) donor-donor $\left(R_{d d}\right)$ and donor-acceptor $\left(R_{d a}\right)$ Förster radii for each vibronic coupling considered here. b) Average and standard deviation of optimal $R_{d d}$ and $R_{d a}$ for scenarios that differ by the acceptor's bandgap with $k_{0}$ fixed at $10^{13} \mathrm{~s}^{-1}$. c) Average and standard deviations of IQEs calculated for different acceptor bandgaps with $k_{0}$ fixed at $10^{13} \mathrm{~s}^{-1}$.

We turn now to the efficiency of exciton diffusion in the various scenarios. To this end, it is necessary to analyze the behavior of the Förster radius in the top ten highest EQE simulations found by the genetic algorithm. As mentioned above, the Förster radius is a characteristic distance at which the exciton is equally likely to recombine radiatively and to hop towards a neighboring molecule. The higher this quantity, the higher the exciton diffusion length is and the more efficient is the diffusion process. In Figure 5-a, we present the average donor-donor $\left(\mathrm{R}_{d d}\right)$ and donor-acceptor $\left(\mathrm{R}_{d a}\right)$ Förster radii for the top ten simulations in the different scenarios along with their standard deviation. The donordonor Förster radius controls the efficiency of exciton diffusion in the bulk of the donor material and its average optimal values decrease from $76 \AA$ for $k_{0}=10^{10}$ $\mathrm{s}^{-1}$ to $63 \AA$ for $k_{0}=10^{14} \mathrm{~s}^{-1}$. The reason for this reduction is due to the larger energy gaps and lower radiative lifetimes selected in the simulations with higher non-radiative rates. Both features reduce the Förster radius, as calculated in Equation 8 . This reduction shortens the exciton diffusion length, explaining the selection of smaller donor domain sizes in the cases of higher internal conversion rates, as we have seen above. Nevertheless, these values of Förster radii can be considered large in comparison to those calculated for typical organic molecules ${ }^{[29]}$. This indicates the algorithm's preference for maximizing the efficiency of exciton diffusion in the donor material, which can be achieved by selecting larger transition dipole moments.

In opposite behavior to the donor-donor Förster radius, the optimal donor-acceptor radius is seen to increase steadily with $k_{0}$. This is also due to the larger donor gaps, which eventually result in the appearance of an overlap between the donor's emission spectrum and the acceptor's absorption spectrum. The increase of the donor-acceptor Förster radius makes it possible for excitons to hop across the interface, constituting a competing phenomenon to exciton dissociation and a loss mechanism. Such competition may be prevented by the proper choice of acceptor gap. In this sense, we consider a set of optimization procedures run with a constant $k_{0}=10^{13} \mathrm{~s}^{-1}$, a fixed acceptor LUMO and varying acceptor energy gaps. This choice is made so we may attribute changes in optimization strategy and efficiency solely on the behavior of the acceptor's absorption peak energy. The effects of this substitution on exciton diffusion are shown in Figure 5-b, where it can be seen that the donor-donor Förster radius is kept constant around $60 \AA$ whereas the donor-acceptor radius steadily decreases. We may evaluate the consequences of the elim- 
ination of this competing phenomenon by comparing the average efficiencies among these different simulations. A $0.3 \%$ increase in average EQE from $5.8 \%$ to $6.1 \%$ is observed when the acceptor gap goes from 2.4 $\mathrm{eV}$ to $2.8 \mathrm{eV}$. Even though this seems as a very small difference, the corresponding IQE rises by almost $10 \%$, as shown in Figure 5-c, in correlation with a drop in donoracceptor Förster radius from $49 \AA$ to $33 \AA$. However, after this point, no further gains in efficiency are observed. This is due to the fact that the Förster transfer rate is proportional to the sixth power of the Förster radius. As such, when $R_{d a}$ changes from $49 \AA$ to $33 \AA$, even though this corresponds to a $33 \%$ decrease, the actual reduction in the rates for transfers across the interface amounts to $90 \%$. Interestingly, the only feature other than $R_{d a}$ that suffers considerable changes in optimization for different acceptor gaps is the electronic coupling $t$, whose average optimal value decreases from $29 \mathrm{meV}$ to $19 \mathrm{meV}$ as shown in Figure SI-2. This suggests that the prevention of Förster transfers at the interface may reduce the device's dependence on large electronic couplings, which are a harder feature to engineer. These observations demonstrate that small tweaks in the acceptor's gap may provide important efficiency gains in terms of exciton dissociation.

All these results allow us to reach some important design principles. First, we conclude that quantum yields are more relevant to device efficiency than exciton lifetimes, so focus should be on the former rather than the latter as given any amount of non-radiative transitions, the optimal configuration moves towards higher quantum yields. Second, better compatibility between donor and acceptor can be reached by both preventing overlap between the donor's emission and the acceptor's absorption spectra and by a choice in LUMO-LUMO energy differences that takes into account the downhill exciton diffusion that results from energetic disorder. As we have seen, the elimination of competing phenomena at the interface associated with proper pairing of frontier energy levels reduces the dependence of exciton dissociation efficiency on electronic couplings and results in larger EQE and IQE.

\section{4 | CONCLUSIONS}

We present a comprehensive model of exciton dynamics from light absorption to exciton dissociation at a donoracceptor interface that is coupled to a genetic algorithm with the objective of finding optimization strategies under different scenarios that differ by the strength of the vibronic coupling of the donor molecule. This approach was able to reproduce some design principles already known and also provided new insights.

In agreement with previous experimental works, we have determined that overall the evolution process pushes towards systems that present donor domain sizes in the range of 10-12 $\mathrm{nm}$ and transition dipole moments around 10 a.u., which are indicative of polymers. When non-radiative decay rates are low, the optimization points at low bandgap polymers as optimal donor materials. As these rates grow larger, the optimal bandgap increases significantly. This corresponds to an attempt at keeping quantum yields high, highlighting the importance of this feature to OPVs as have been stressed by recent works. The increase in quantum yields comes with a simultaneous reduction of exciton radiative lifetimes and non-radiative rates, which results from increased bandgaps. In addition, we find that the pairing of donor-acceptor LUMOs must take into account the effects of energetic disorder on exciton diffusion so that dissociation rates at the interface can be maximized. Such disorder is required to be kept in check by selecting spectral broadness given by a standard deviation near $0.17 \mathrm{eV}$.

Exciton diffusion plays an important role, with the optimization process selecting large donor-donor Förster radii, which are associated with longer diffusion lengths. This feature correlates with the donor domain size, meaning that increases in this radius allows for larger donor domains to be employed which result in more efficient light harvesting. Large donor-acceptor Förster radii, on the other hand, constitute a loss mechanism, and its elimination is determined to increase both EQE and IQE as well as reduce the device's dependence on large electronic couplings, which are more difficult to engineer. This latter feature, however, is often overlooked 
when considering device design. These results provide several principles for the rational design of devices and the computational protocol shown here also constitutes a promising method for optimizing the pairing of donor and acceptor molecules for photovoltaic applications.

\section{Acknowledgements}

This study was financed in part by the Coordenacao de Aperfeicoamento de Pessoal de Nivel Superior Brasil (CAPES) - Codigo de Financiamento 001. The authors also acknowledge the support from CNPq and FAP-DF. D.A.S.F acknowledges the support of the Institute of Advanced Studies of the Université de CergyPontoise under the Paris Seine Initiative for Excellence ("Investissements Avenir" ANR-16-IDEX-0008) and the financial support from the Edital DPI - UnB Nº 04/2019, from CNPq (grants 304020/2016-8, 305975/20196 and 420836/2018-7) and FAP-DF (grants 193.001. $596 / 2017$ and 193.001.284/2016).

\section{Conflict of Interest}

There are no conflicts of interest to report.

\section{Supporting Information}

Supporting information is available.

\section{References}

1. Lin X Chen, Organic Solar Cells: Recent Progress and Challenges, 2019.

2. Veaceslav Coropceanu, Xian-Kai Chen, Tonghui Wang, Zilong Zheng, Jean-Luc Brédas, Nature Reviews Materials 2019, 1-19.

3. Swaminathan Venkatesan, Evan Ngo, Devendra Khatiwada, Cheng Zhang, Qiquan Qiao, ACS applied materials \& interfaces 2015, 7 (29), 16093-16100.

4. Deping Qian, Zilong Zheng, Huifeng Yao, Wolfgang Tress, Thomas R Hopper, Shula Chen, Sunsun Li, Jing Liu, Shangshang Chen, Jiangbin Zhang, et al., Nature materials 2018, 17 (8), 703.
5. Zilong Zheng, Naga Rajesh Tummala, Tonghui Wang, Veaceslav Coropceanu, Jean-Luc Brédas, Advanced Energy Materials 2019, 9 (14), 1803926.

6. Jean-Luc Brédas, Joseph E Norton, Jérôme Cornil, Veaceslav Coropceanu, Accounts of chemical research 2009, 42 (11), 1691-1699.

7. Bernard Kippelen, Jean-Luc Brédas, Energy \& Environmental Science 2009, 2 (3), 251-261.

8. Th Förster, Annalen der physik 1948, 437 (1-2), 55-75.

9. Th Förster, Discussions of the Faraday Society 1959, 27, 7-17.

10. Volkhard May, K Oliver, Charge and energy transfer $d y$ namics in molecular systems, John Wiley \& Sons, 2008.

11. Robert Englman, Joshua Jortner, Molecular Physics 1970, 18 (2), 145-164.

12. Stoichko Dimitrov, Bob Schroeder, Christian Nielsen, Hugo Bronstein, Zhuping Fei, lain McCulloch, Martin Heeney, James Durrant, Polymers 2016, 8 (1), 14.

13. Johannes Benduhn, Kristofer Tvingstedt, Fortunato Piersimoni, Sascha Ullbrich, Yeli Fan, Manuel Tropiano, Kathryn A McGarry, Olaf Zeika, Moritz K Riede, Christopher J Douglas, et al., Nature Energy 2017, 2 (6), 1-6.

14. Veaceslav Coropceanu, Jérôme Cornil, Demetrio A da Silva Filho, Yoann Olivier, Robert Silbey, Jean-Luc Brédas, Chemical reviews 2007, 107 (4), 926-952.

15. Tianyu Zhu, Troy Van Voorhis, Piotr de Silva in Handbook of Materials Modeling, Springer, 2018.

16. Markus C Scharber, David Mühlbacher, Markus Koppe, Patrick Denk, Christoph Waldauf, Alan J Heeger, Christoph J Brabec, Advanced materials 2006, 18 (6), 789-794.

17. Lal Mohammad, Abu Farzan Mitul, Sudhan Sigdel, Ashish Dubey, Devendra Khatiwada, Nirmal Adhikari, Hytham Elbohy, Qiquan Qiao, IEEE Journal of Photovoltaics 2015, 5 (6), 1659-1664.

18. Moneim Elshobaki, Ryan Gebhardt, John Carr, William Lindemann, Wenjie Wang, Eric Grieser, Swaminathan Venkatesan, Evan Ngo, Ujjal Bhattacharjee, Joseph Strzalka, et al., ACS applied materials \& interfaces 2017, 9 (3), 2678-2685. 
19. Devendra Khatiwada, Swaminathan Venkatesan, Evan C Ngo, Qiquan Qiao, Journal of nanoscience and nanotechnology 2015, 15 (9), 7040-7044.

20. Lal Mohammad, Abu Mitul, Qi Wang, Swaminathan Venkatesan, Devendra Khatiwada, Ashish Dubey, Cheuk-Lam Ho, Wai-Yeung Wong, Qiquan Qiao, IEEE Transactions on Electron Devices 2015, 62 (9), 30293033.

21. Devendra Khatiwada, Swaminathan Venkatesan, Qiliang Chen, Jihua Chen, Nirmal Adhikari, Ashish Dubey, Abu Farzan Mitul, Lal Mohammed, Qiquan Qiao, Journal of Materials Chemistry A 2015, 3 (29), 1530715313.

22. Yilei Wu, Sebastian Schneider, Christopher Walter, Ashraful Haider Chowdhury, Behzad Bahrami, HungChin Wu, Qiquan Qiao, Michael F Toney, Zhenan Bao, Journal of the American Chemical Society 2019.

23. Swaminathan Venkatesan, Jianyuan Sun, Lianjie Zhang, Ashish Dubey, Andrew Sykes, Ting-Yu Lin, Yu-Chueh Hung, Qiquan Qiao, Cheng Zhang, RSC advances 2016, 6 (104), 102043-102056.

24. Leonardo Evaristo de Sousa, Luiz Antonio Ribeiro, Antonio Luciano de Almeida Fonseca, Demetrio Antonio da Silva Filho, The Journal of Physical Chemistry A 2016, 120 (27), 5380-5388.

25. Igo T Lima, Andriele da S Prado, Joao BL Martins, Pedro Henrique de Oliveira Neto, Artemis M Ceschin, Wiliam F da Cunha, Demetrio A da Silva Filho, The Journal of Physical Chemistry A 2016, 120 (27), 4944-4950.

26. Rachel Crespo-Otero, Mario Barbatti, Theoretical Chemistry Accounts 2012, 131 (6), 1237.

27. Daniel FS Machado, Thiago O Lopes, Igo T Lima, Demetrio A da Silva Filho, Heibbe CB de Oliveira, The Journal of Physical Chemistry C 2016, 120 (31), 1766017669.

28. Josiah A Bjorgaard, Muhammet Erkan Köse, Rsc Advances 2015, 5 (11), 8432-8445.

29. Leonardo Evaristo de Sousa, Fernando Teixeira Bueno, Geraldo Magela e Silva, Demétrio Antônio da Silva Filho, Pedro Henrique de Oliveira Neto, Journal of Materials Chemistry C 2019, 7 (14), 4066-4071.

30. Conor Madigan, Vladimir Bulović, Physical review letters 2006, 96 (4), 046404.
31. Leonardo Evaristo de Sousa, Demétrio Antônio da Silva Filho, Rafael Timóteo de Sousa, Pedro Henrique de Oliveira Neto, Scientific reports 2018, 8 (1), 14066.

32. Hiroyuki Tamura, Koki Azumaya, Hiroshi Ishikita, The journal of physical chemistry letters 2019, 10 (24), 76237628.

33. Leonardo Evaristo de Sousa, Fernando Teixeira Bueno, Luciano Ribeiro, Luiz Antonio Ribeiro Junior, Demetrio Antonio da Silva Filho, Pedro Henrique de Oliveira Neto, Chemistry of Materials 2019, 31 (17), 6818-6823.

34. Heinz Bässler, physica status solidi (b) 1993, 175 (1), 1556.

35. Riccardo Volpi, Sathish Kottravel, Morten Steen Nørby, Sven Stafstrom, Mathieu Linares, Journal of chemical theory and computation 2016, 12 (2), 812-824.

36. Haoyuan Li, Gjergi Sini, Joseph Sit, Adam Moule, JeanLuc Bredas, Energy \& Environmental Science 2020.

37. Veaceslav Coropceanu, Jean-Luc Bredas, Shafigh Mehraeen, The Journal of Physical Chemistry C 2017, 121 (45), 24954-24961.

38. Jakob Kjelstrup-Hansen, Joseph E Norton, Demetrio A da Silva Filho, Jean-Luc Brédas, Horst-Günter Rubahn, Organic Electronics 2009, 10 (7), 1228-1234.

39. Upendra Neupane, Behzad Bahrami, Matt Biesecker, Mahdi Farrokh Baroughi, Qiquan Qiao, Nano energy 2017, 35, 128-137.

40. Kim F Wong, Biman Bagchi, Peter J Rossky, The Journal of Physical Chemistry A 2004, 108 (27), 5752-5763.

41. Pavel Moroz, Zhicheng Jin, Yuya Sugiyama, D'Andree Lara, Natalia Razgoniaeva, Mingrui Yang, Natalia Kholmicheva, Dmitriy Khon, Hedi Mattoussi, Mikhail Zamkov, ACS nano 2018, 12 (6), 5657-5665.

42. Michael H Stewart, Alan L Huston, Amy M Scott, Eunkeu Oh, W Russ Algar, Jeffrey R Deschamps, Kimihiro Susumu, Vaibhav Jain, Duane E Prasuhn, Juan Blanco-Canosa, et al., ACS nano 2013, 7 (10), 94899505.

43. Dominique Douguet, Etienne Thoreau, Gérard Grassy, Journal of computer-aided molecular design 2000, 14 (5), 449-466. 
44. Maxim Tafipolsky, Rochus Schmid, The Journal of Physical Chemistry B 2009, 113 (5), 1341-1352.

45. Luiz Antonio Ribeiro Jr, Pedro Henrique de Oliveira Neto, Wiliam Ferreira da Cunha, Ricardo Gargano, Geraldo Magela e Silva, Chemical Physics Letters 2013, 555, 168-172.

46. Leonardo Evaristo de Sousa, Pedro Henrique de Oliveira Neto, Jakob Kjelstrup-Hansen, Demétrio Antônio da Silva Filho, The Journal of chemical physics 2018, 148 (20), 204101.

47. Piotr de Silva, Troy Van Voorhis, The journal of physical chemistry letters 2018, 9 (6), 1329-1334.

48. David J Tannor, Eric J Heller, The Journal of Chemical Physics 1982, 77 (1), 202-218.

49. Edward L Mertz, Vyacheslav A Tikhomirov, Lev I Krishtalik, The Journal of Physical Chemistry A 1997, 101 (19), 3433-3442.

50. Wolfgang Tress in Organic Solar Cells, Springer, 2014, pp. 67-214.

51. Bijan Movaghar, M Grünewald, B Ries, H Bassler, D Würtz, Physical Review B 1986, 33 (8), 5545.

52. Shane R Yost, Eric Hontz, Sina Yeganeh, Troy Van Voorhis, The Journal of Physical Chemistry C 2012, 116 (33), 17369-17377.

53. Anna Köhler, Heinz Bässler, Electronic processes in organic semiconductors: An introduction, John Wiley \& Sons, 2015.

54. RA Marcus, The Journal of Physical Chemistry 1989, 93 (8), 3078-3086.

55. Martin Knupfer, Applied Physics A 2003, 77 (5), 623626.

56. Demetrio A da Silva Filho, Veaceslav Coropceanu, Nadine E Gruhn, Pedro Henrique de Oliveira Neto, JeanLuc Brédas, Chemical Communications 2013, 49 (54), 6069-6071.

57. Wanli Ma, Cuiying Yang, Xiong Gong, Kwanghee Lee, Alan J Heeger, Advanced Functional Materials 2005, 15 (10), 1617-1622.

58. Gang Yu, Jun Gao, Jan C Hummelen, Fred Wudl, Alan J Heeger, Science 1995, 270 (5243), 1789-1791.

59. Barry C Thompson, Jean MJ Fréchet, Angewandte chemie international edition 2008, 47 (1), 58-77.
60. Fiona C Jamieson, Ester Buchaca Domingo, Thomas McCarthy-Ward, Martin Heeney, Natalie Stingelin, James R Durrant, Chemical Science 2012, 3 (2), 485492.

61. Carsten Deibel, Vladimir Dyakonov, Christoph J Brabec, IEEE Journal of Selected Topics in Quantum Electronics 2010, 16 (6), 1517-1527.

62. Obaid Alqahtani, Maxime Babics, Julien Gorenflot, Victoria Savikhin, Thomas Ferron, Ahmed H Balawi, Andreas Paulke, Zhipeng Kan, Michael Pope, Andrew J Clulow, et al., Advanced Energy Materials 2018, 8 (19), 1702941.

63. Subhrangsu Mukherjee, Christopher M Proctor, John R Tumbleston, Guillermo C Bazan, Thuc-Quyen Nguyen, Harald Ade, Advanced Materials 2015, 27 (6), 11051111.

64. Jianchen $\mathrm{Hu}$, Yasuhiro Shirai, Liyuan Han, Yutaka Wakayama, Nanoscale research letters 2012, 7 (1), 469.

65. Christos L Chochos, Athanasios Katsouras, Nicola Gasparini, Chrysanthos Koulogiannis, Tayebeh Ameri, Christoph J Brabec, Apostolos Avgeropoulos, Macromolecular rapid communications 2017, 38 (2), 1600614.

66. Eva Bundgaard, Frederik C Krebs, Solar Energy Materials and Solar Cells 2007, 91 (11), 954-985.

67. Kedar D Deshmukh, Tianshi Qin, Joseph K Gallaher, Amelia CY Liu, Eliot Gann, Kane O'Donnell, Lars Thomsen, Justin M Hodgkiss, Scott E Watkins, Christopher R McNeill, Energy \& Environmental Science 2015, 8 (1), 332-342.

68. Chuanfei Wang, Xiaofeng Xu, Wei Zhang, Jonas Bergqvist, Yuxin Xia, Xiangyi Meng, Kim Bini, Wei Ma, Arkady Yartsev, Koen Vandewal, et al., Advanced Energy Materials 2016, 6 (18), 1600148.

69. Daize Mo, Huan Wang, Hui Chen, Shiwei Qu, Pengjie Chao, Zhen Yang, Leilei Tian, Yu-An Su, Yu Gao, Bing Yang, et al., Chemistry of Materials 2017, 29 (7), 28192830.

70. Zhi-Guo Zhang, Yankang Yang, Jia Yao, Lingwei Xue, Shanshan Chen, Xiaojun Li, William Morrison, Changduk Yang, Yongfang Li, Angewandte Chemie International Edition 2017, 56 (43), 13503-13507.

71. Chang Liu, Kai Wang, Xiong Gong, Alan J Heeger, Chemical Society Reviews 2016, 45 (17), 4825-4846. 
72. Gang Li, Wei-Hsuan Chang, Yang Yang, Nature Reviews Materials 2017, 2 (8), 17043.

73. Letian Dou, Yongsheng Liu, Ziruo Hong, Gang Li, Yang Yang, Chemical reviews 2015, 115 (23), 12633-12665.

74. Jun Yuan, Tianyi Huang, Pei Cheng, Yingping Zou, Huotian Zhang, Jonathan Lee Yang, Sheng-Yung Chang, Zhenzhen Zhang, Wenchao Huang, Rui Wang, et al., Nature communications 2019, 10 (1), 1-8. 